\title{
ARTESANOS EMPRENDEDORES DE LA ARTESANÍA LOCAL EN LA PROVINCIA DE GRANMA, CUBA
}

\author{
D. RAMÍREZ ${ }^{1}$, S. RODRÍGUEZ ${ }^{1}$, A. N. CUTIÑO ${ }^{1}$, E. LEITE ${ }^{2}$, Y. PÉREZ ${ }^{1}$, C. VALDESPINO ${ }^{1 *}$, M. C. CONCI ${ }^{3}$, G. CRUPI $^{4}$, B. \\ MOINE ${ }^{3}$, Y. RAMOS ${ }^{1}$, I. ARIAS ${ }^{1}$, D. TAMAYO ${ }^{1}$, A. ALMAGUER ${ }^{6}$, K. ESCALONA $^{7}$, K. FERNÁNDEZ $^{1}$, L. FIGUEREDO e \\ M. GIL ${ }^{1}$ \\ ${ }^{1}$ Universidad de Granma. Cuba \\ ${ }^{2}$ Universidad de Pernambuco. Brasil \\ ${ }^{3}$ Universidad Nacional de Villa María. Argentina \\ ${ }^{4}$ Universidad Nacional del Nordeste de la Provincia de Buenos Aires. Argentina, SENESCYT. Ecuador \\ ${ }^{6}$ Universidad de las Américas, Ecuador \\ cvaldespinot@udg.co.cu*
}

Artículo recibido en en marzo/2016 y aceptado en abril/2016

DOI: $10.15628 /$ holos.2016.4323

\section{RESUMEN}

La provincia Granma se encuentra ubicada en la región suroriental de Cuba. Su riqueza natural constituye uno de sus principales atractivos, al mismo tiempo que proporciona la materia prima fundamental para desarrollar una de las expresiones culturales que más fuerzas ha adquirido en los últimos años, la artesanía. El uso racional de productos naturales constituye actualmente el soporte básico para la creación y desarrollo de objetos artesanales, los cuales son representativos de la cubanía e identidad nacional, así como portadores de utilidad práctica y de valores decorativos. Las industrias culturales se clasifican en nueve grupos según el Observatorio de Cultura y Economía (2011), en la cual se reconoce la artesanía, por lo tanto es un motor impulsor de la cultura y de las tradiciones. El estudio del fenómeno del emprendimiento se considera necesario para obtener una obra auténtica, por lo tanto en este artículo se abordan algunos aspectos que se consideran relevantes en torno al emprendimiento. El presente trabajo tiene como objetivo general analizar el comportamiento de la artesanía y el fenómeno del emprendimiento en la provincia Granma, como elemento dinamizador de la industria cultural. Se analiza una muestra de artesanos tomados al azar a través del método estadístico Análisis de Correspondencia Simple, el cual arrojó resultados muy interesantes relacionados con las variables capacitación, motivación e innovación. Finalmente se propone un plan de acciones para fomentar el desarrollo artesanal en la provincia Granma.

PALABRAS-CLAVE: artesanía, emprendimiento, identidad, industria cultural, desarrollo local.

\section{ENTREPRENEURS OF THE LOCAL ARTISANS HANDICRAFTS IN THE PROVINCE OF GRANMA, CUBA}

\begin{abstract}
Granma province is located in the south-eastern region of Cuba. Its natural wealth is one of its main attractions, while providing the basis for developing a cultural expression that has gained more strength in recent years, handicraft raw material. The rational use of natural products is currently the basic support for the creation and development of handicrafts, which are representative of the Cuban identity and national identity, as well as having practical utility and decorative values. Cultural industries are classified into nine groups according to the Observatory of Culture and Economics (2011), in which the craft is recognized, therefore it is a driving force of culture and traditions. The study of the
\end{abstract}

phenomenon of entrepreneurship is considered necessary for a real work, so in this article some aspects considered relevant about entrepreneurship are addressed. The present work has as general objective to analyze the behavior of the craft and the phenomenon of entrepreneurship in Granma province, as a catalyst for the cultural industry. A random sample of artisans was taken through the Simple Correspondence Analysis statistical method, which yielded very interesting resultsrelated to the training, motivation and innovation variables. Finally an action plan aims to encourage craft development in Granma province was proposed.

KEYWORDS: handicrafts, entrepreneurship, identity, cultural industry, local development. 


\section{INTRODUCCÍON}

El presente estudio tiene como objetivo general analizar el comportamiento de la artesanía y el fenómeno del emprendimiento en la provincia Granma, como elementos dinamizadores de la industria cultural. La provincia Granma, se ubica en la región sur-oriental de Cuba. Los municipios Bayamo y Manzanillo son los más representativos en el desarrollo de actividades artesanales. Sin embargo no son aprovechadas al máximo todas las potencialidades naturales de la región como fuente primaria de materia prima para la elaboración de los disímiles objetos de uso estético, utilitario e identitario que pueden resultar de un manejo eficiente y racional de elementos naturales para ello.

Las industrias culturales han alcanzado un papel muy importante en la preservación de la cultura e identidad nacional. Se definen como una combinación de producción y comercialización de contenidos que son tangibles e intangibles y que al mismo tiempo son culturales en su naturaleza, considerando que están protegidos por el derecho de autor y pueden tomar forma de bienes o servicios. (Piedras, 1994).

La clasificación de las Industrias Culturales según el OBSERVATORIO CULTURA Y ECONOMÍA (2011) está dada en nueve grupos, los cuales comprenden: artes escénicas(música, danza, marionetas, circo y ópera), audiovisuales (cine, TV y radio), servicios creativos (arquitectura, publicidad), media interactiva (contenidos digitales, software, video juegos, animaciones), diseños interiores (gráficos, modas y joyería), literatura y publicaciones (libros, prensa y otras publicaciones), artes visuales (pintura, escultura, fotografía y antigüedades), patrimonio cultural (museos, bibliotecas, sitios arqueológicos) y conocimiento tradicional (festivales, artesanías).La artesanía como exponente cultural de una región o localidad requiere tanto como el cine, el teatro, la danza, las artes plásticas, el apoyo gubernamental, así como la capacitación de los involucrados en la actividad. Se necesita desarrollar una mayor conciencia social del valor cultural que reporta, así como del socioeconómico.

El artesano es capaz de conformar a través de sus manos un objeto que refleje tradiciones, identidad, historia, valores naturales y patrimoniales, costumbres, o que sea capaz de satisfacer una determinada necesidad social, es por eso que se considera muy importante la presente investigación donde se analiza la artesanía desde una mirada cultural, y a la vez se abordan los postulados principales del fenómeno del emprendimiento, para lograr que la artesanía sea exponente auténtico de la cultura local.

\section{REVISIÓN BIBLIOGRÁFICA}

A mediados de los años cuarenta se utilizó el término de industria cultural, para explicar un cambio en el proceso de transmisión de la cultura, donde se hace referencia a la radio, el cine y la naciente televisión (Horkheimer y Adorno, 1949).

Zallo (1988) se refiere a las industrias culturales como el conjunto de ramas industriales, productoras y distribuidoras de mercancías con contenido simbólico, concebidas gracias a un trabajo donde interviene la creatividad e imaginación, organizadas por un capital que se valoriza y destinadas a un mercado de consumo, con fines de producción ideológica y social. 
Las industrias culturales han tomado un gran auge a nivel internacional, según estudios internacionales desarrollados por la ONU en 1998 acerca de las aportaciones económicas derivadas de la industria cultural en Europa. Por ejemplo, las industrias culturales generan el 4,5\% del PIB europeo, además de que se valora en 1800 millones de euros los que la Unión Europea destinará a la financiación de proyectos culturales en el periodo 2014-2020 (Banco Santander, 2012).

De acuerdo con lo planteado por (Bouchart, 2004) en cuanto al ámbito de la producción artesanal como parte de las industrias culturales, algunos países industrializados otorgan mucha importancia a las artesanías, un ejemplo de ello se encuentra en Italia, que en el año 2001 estimó que el $24 \%$ de sus empresas eran artesanales, además de que la exportación de las artesanías representaba el $17 \%$ del PIB. En Colombia la producción de objetos artesanales produjo unos 400 millones de dólares, además de que las exportaciones de esos objetos ascendieron a 40 millones de dólares. México, por su parte, de acuerdo con el FONART, contaba con 2,8 millones de artesanos de tiempo completo, además de 4 millones más de tiempo parcial, lo que hace un total de 6.8 millones de artesanos que operaban en el país.

El artesano, tal como lo conocemos actualmente, tiene su origen en las sociedades europeas, donde se desarrollaron como una parte de la sociedad que se especializaba en diferentes oficios, con el fin de producir tanto objetos cotidianos como de lujo, para el resto de la sociedad.

Según (Throsby, 2011) el concepto de artesanía supone lo siguiente:

"Los productos artesanales son los producidos por artesanos, ya sea totalmente a mano, o con la ayuda de herramientas manuales o incluso de medios mecánicos, siempre que la contribución manual directa del artesano siga siendo el componente más importante del producto acabado" (...).

Novelo (1993), hace referencia al término artesanal como una peculiar manera en la que el hombre ha usado los instrumentos de trabajo para producir algo, generalmente un bien o un objeto de consumo.

Pasteur (2004) menciona que las artesanías tradicionales son aquellos bienes realizados para el consumo doméstico y/o ritual, siendo objetos portadores de un valor histórico, cultural, utilitario o estético y que cumplen con una función socialmente reconocida, realizados por el artesano, individual o colectivamente, mediante técnicas simples.

Como ya se mencionó anteriormente tanto Europa como América Latina son portadoras de este arte milenario, aportándoles importantes ingresos a sus economías. En el caso particular de Cuba, un país multicultural, con una enorme riqueza en cuanto al patrimonio arqueológico, histórico y artístico, la artesanía es asumida como parte inseparable de su acervo cultural, es un arte ancestral. Sus orígenes se remontan a la comunidad aborigen que poblaron estas tierras. Aunque sin tener plena conciencia del tipo de actividad que desarrollaban, de estos grupos surgen las primeras manifestaciones artesanales.

La Asociación Cubana de Artesanos Artistas (ACAA), que agrupa al gremio de artesanos del país, con filiales en todas las provincias, tiene como principal objetivo alcanzar el máximo desarrollo de la artesanía nacional.

En la provincia Granma, el ACCA constituye una de las principales fortalezas que pueden dinamizar esta actividad, como institución legal que aglutina, dirige, asesora y controla todo lo 
referente a la artesanía. También representan potencialidades los materiales más disímiles con los que se pueden elaborar estos productos, una gran parte de ellos de fácil acceso dentro de los límites geográficos de la provincia. El mármol es uno de los productos accesibles para el trabajo artesanal, con este recurso se pueden elaborar desde objetos utilitarios hasta decorativos. También la naturaleza, quien representa la principal fuente de materia prima, aporta el coco, cuyo recurso es muy utilizado para elaborar prendas tanto femeninas como masculinas, objetos decorativos, musicales, entre otros. El barro, es también muy utilizado, fundamentalmente para elaborar figuras y objetos de utilidad en el hogar. El bejuco y el bambú, son también importantes para elaborar bolsos, muebles, cestas, mesas e incluso casas de descanso en el campo.

La artesanía puede constituir una estrategia alternativa para el desarrollo local. En primera instancia se considera necesario que los artesanos tomen conciencia del incalculable valor de sus obras desde el punto de vista inmaterial, pues estás deberán ser un reflejo auténtico de la cubanía que caracteriza la región. La autenticidad y creatividad de las obras son una premisa fundamental para lograr posicionar este sector. Por otro lado se debe ver como un factor clave en el desarrollo local, pues aprovecha las potencialidades endógenas del territorio, materializándose a través de productos que son representativos de la cultura. En una región prácticamente virgen en esta área, su fomento no solo repercute en la satisfacción de los habitantes de la localidad, sino que contribuiría a impulsar el turismo cultural en la zona, lo que a su vez tiene una influencia directa en la economía local.

Estas obras no solo son portadoras de significado simbólico sino que reflejan sentimientos, lo cual las hace de difícil valoración económica y en segundo lugar el conocimiento tradicional de los artesanos, que es un recurso y una capacidad incuantificable. (Vargas, 2013)

La artesanía presenta algunas debilidades vinculadas a una idea confusa y distorsionada sobre este sector. Hay quienes se acercan a la artesanía buscando gangas, sin considerar que se trata de trabajos únicos, salidos de talleres de auténticos profesionales. "La artesanía ofrece una clara resistencia a su industrialización, lo que hace que haya un percepción de lo artesanal como una actividad obsoleta y la percepción que tienen las administraciones públicas y los agentes que prestan servicios a las empresas (las entidades financieras, entre otras) de que la artesanía es una actividad informal, sin peso económico, es una pura utopía (Ramos, 2009).

La UNESCO es la única organización internacional con una visión global del papel social, económico y cultural de la artesanía en la sociedad. Por lo que se ha dedicado a desarrollar proyectos relacionados al impulso artesanal, con el objetivo de demostrar a las autoridades de diferentes naciones, estados y localidades, la prioridad que merece la artesanía en los programas de desarrollo. (Vargas, 2013)

Por otra parte, es necesario abordar también el rol que juega el espíritu emprendedor en el oficio del artesano. Un emprendedor es un individuo que se lanza a nuevos proyectos, es creativo, arrojado y busca hacer actividades diferentes. Los emprendedores poseen características peculiares basadas en la perspectiva de que la creatividad no depende de la inspiración, sino de un arduo estudio y un acto de voluntad.

Los emprendedores artesanos son aquellos que crean empresas para trabajar en ellas y no para especular con ellas. Enfocan su trabajo entendiendo a que los objetos y servicios que se ofrecen en el mercado son portadores de proyectos sociales y visiones morales y en su proyecto 
empresarial generan inherentemente el bienestar social, producto de todo proyecto empresarial sostenible y honesto (DE UGARTE, 2009).

Los emprendedores están involucrados en un proceso que el economista (Schumpeter, 1984 y 1988) describió como "destrucción creativa", que consiste en romper con viejos hábitos con el instinto de generar nuevas respuestas a las carencias y deseos del mercado.

La finalidad de la actividad emprendedora es la satisfacción de los deseos y necesidades de los individuos, materializados por la obtención de ganancias. La satisfacción del cliente es el combustible de la acción emprendedora, es lo que impulsa al emprendedor a avanzar cada vez más en la lucha por la supervivencia y por el crecimiento de la iniciativa.

Bajo la óptica schumpeteriana, la innovación permite, generalmente, la construcción de nuevas organizaciones o, por lo menos, una transformación radical en las antiguas organizaciones. La creación de nuevas organizaciones puede darse con el nacimiento de nuevas empresas o la ampliación de antiguas. El mundo de los negocios es muy dinámico y las fuerzas del mercado están en constante mutación.

(Mcclelland, 1961 y 1987), define al emprendedor como alguien que ejerce cierto control sobre los medios de distribución y producen más de lo que puede consumir, con el objetivo de venderlo (o cambiarlo) para lograr una renta individual o doméstica.

Características del artesano emprendedor

- Las principales características del comportamiento emprendedor de los artesanos son las siguientes:

- Búsqueda de oportunidad e iniciativa

- Persistencia

- Exigencia de calidad, eficiencia y eficacia

- Comprometimiento

- Búsqueda de informaciones

- Establecimiento de metas

- Planificación y monitoreo sistemáticos

- Persistencia y redes de contacto

- Independencia e autoconfianza

\section{METOdOLOGÍA}

Se seleccionaron personas que se dedican al oficio de artesanos en los dos municipios de la provincia Granma con más desarrollo en la actividad, Bayamo y Manzanillo. Se agruparon de la siguiente forma:

1. Mujeres artesanas comprendidas entre los 20 y 30 años de edad.

2. Hombres artesanos comprendidos entre los 20 y 30 años de edad.

3. Mujeres artesanas comprendidas entre los 30 y 40 años de edad. 
4. Hombres artesanos comprendidos entre los 30 y 40 años de edad.

5. Mujeres artesanas mayores de 40 años de edad.

6. Hombres artesanos mayores de 40 años de edad.

De cada uno de los seis grupos seleccionados se escogieron al azar 10 personas, para una muestra total de 60 artesanos encuestados.

Se aplicó una encuesta que comprendió la siguiente información:

1. ¿Cómo llegó a ser artesano?. Por capacitación (Scap y NCap), por motivación (SMotiv y NMotiv) o por embullo (SEmb y NEmb).

2. ¿Conoce el emprendedorismo? (Conoce Emp y No Conoce Emp).

3. ¿Es una persona emprendedora? (SEmp y NEmp).

4. ¿Es una persona innovadora? (SInnov y NInnov).

5. Barreras que lo limitan ser más emprendedor: Económicas (Barrera Econ); Capacitación (Barrera Cap).

Una vez aplicada y completada la encuesta con la información, se confeccionó una tabla de contingencia con las variables que aparecen entre paréntesis a continuación de las cinco preguntas de la encuesta anteriormente descritas. La información se procesó estadísticamente a través de una Análisis de Correspondencia Simple (Greenacre, 1984, 1988, 1994; Lebart ET AL, 1984), con el empleo del paquete estadístico (Infostatversion libre, 2013). De cada eje se determinó los autovalores y la contribución de cada uno a la inercia o varianza explicada contenida en cada eje, a la Chi cuadrado y a la importancia relativa porcentual de cada uno. Los resultados se muestran a través de una representación gráfica biplot (Gabriel, 1971).

\section{RESULTADOS Y DISCUSIÓN}

En la figura 1 se visualizan las dos primeras dimensiones del Análisis de Correspondencia Simple de la tabla de contingencia correspondiente al cruce de las variables seleccionadas. El gráfico sugiere, en su primer eje, con una inercia de 38,95 \% (Tabla 2), que las mujeres y los hombres artesanos encuestados con una edad superior a los 40 años fueron el grupo de artesanos que menor conocimiento mostraron acerca del emprendimiento, lo que parece influir en que no sean artesanos emprendedores, se iniciaron en este oficio por embullo o estimulación de otras personas que pudieron ser artesanos o no; sin embargo se mostraron como el grupo más innovador; pero el menos motivado y con menor capacitación recibida, constituyendo esta última limitación la principal barrera para ser personas emprendedoras.

El primer eje con un autovalor de 0,76 , contribuye más a la Chi cuadrado, una inercia de 0,58 explica el 38,95 por ciento de la variabilidad total del análisis. El segundo eje con un autovalor de 0,63 , una menor inercia de 0,39 con relación al primer eje y un 26,08 por ciento de la variabilidad total. Entre los dos ejes se explica el 65,08 por ciento de la variación total. Las mujeres y hombres artesanos comprendidos entre los 30 y 40 años de edad fueron el grupo de artesanos que mostró la mayor motivación. 


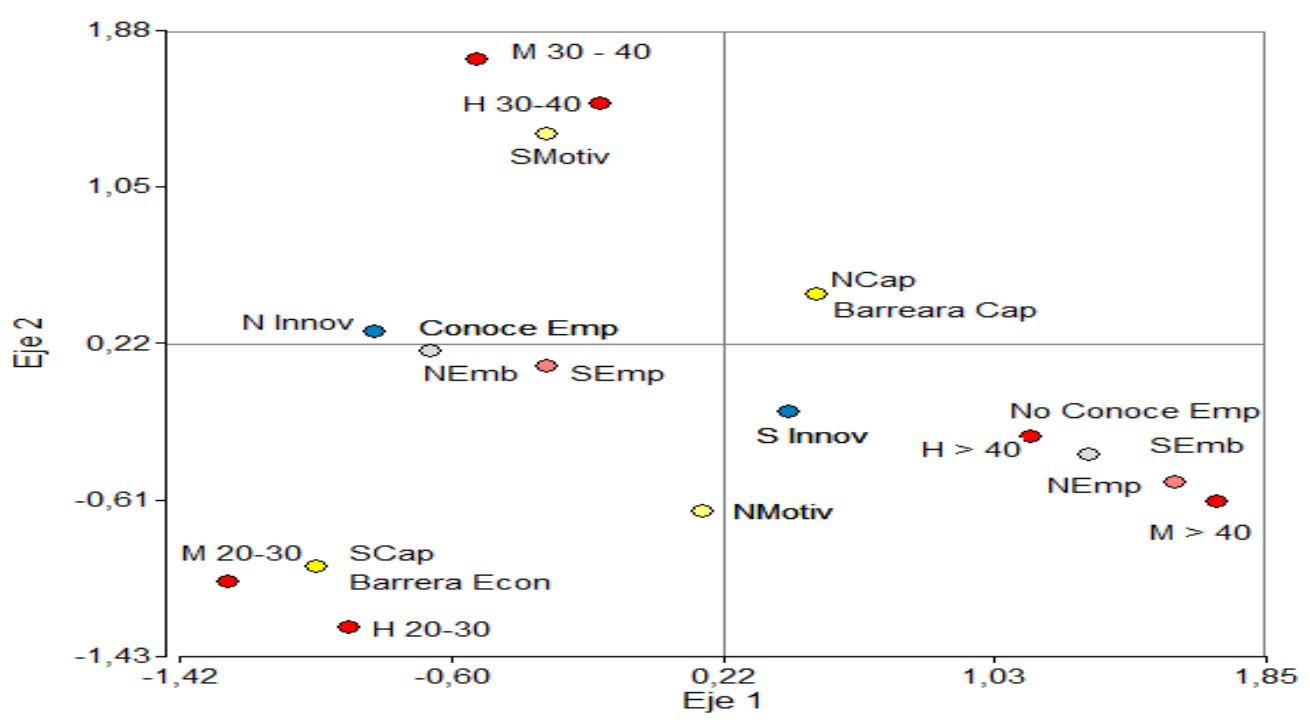

Figura 1: Representación Biplot de los dos primeras componentes del Análisis de Correspondencia Simple de la tabla de contingencia correspondiente al cruce de las variables seleccionadas.

Tabla 2: Contribución a la Chi Cuadrado en el Análisis de Correspondencia Simple

\begin{tabular}{|l|l|l|l|l|l|}
\hline Componente & Autovalor & Inercias & $\begin{array}{l}\text { Chi- } \\
\text { Cuadrado }\end{array}$ & $\%$ & $\begin{array}{l}\% \\
\text { Acumulado }\end{array}$ \\
\hline 1 & 0,76 & 0,58 & 264,83 & 38,95 & 38,95 \\
\hline 2 & 0,63 & 0,39 & 177,35 & 26,08 & 65,03 \\
\hline
\end{tabular}

El grupo de artesanos de ambos sexos con menor edad entre los encuestados correspondientes a las edades comprendidas entre los 20 y 30 años de edad, fue el grupo que se inició en este oficio gracias a la capacitación recibida y no por embullo; pero el factor económico constituyó la principal barrera. Si se comparan con los otros restantes dos grupos, las mujeres y hombres más jóvenes mostraron una mayor inclinación a ser personas emprendedoras, al parecer por poseer conocimientos básicos aunque incipientes acerca del emprendimiento; sin embargo se mostraron como el grupo menos innovador. Sobre la temática de emprendimiento e innovación es importante señalar la amplitud de los trabajos realizados por (Drucker, 1985) y (Leite, 2012 y 2015).

Es de destacar que en el gráfico se observó una clara asociación por grupo de edades, independientemente de que sea mujer $u$ hombre, lo que indica una tendencia entre los artesanos entrevistados a una similitud con relación a los intereses mostrados en la encuesta, más por grupos de edades, que por sexo, mostrándose visiblemente agrupados las mujeres y los hombres entre 20 y 30 años como un grupo, las mujeres y los hombres entre 30 y 40 años como un segundo grupo y un tercer grupo con las mujeres y los hombres mayores de 40 años.

Se puede mencionar como ejemplo el caso de Aldaz y Eguía (2016) que realizaron un estudio bidimensional de la segregación laboral para España y para el País Vasco, considerando el género y la edad como fuentes de desigualdad. Analizaron las posibles diferencias por género en función de la cohorte de edad, así como de estudiar su evolución temporal. Para ello utilizaron microdatos de la Encuesta de Población Activa para los años 2002 y 2014, los representaron a través de curvas de segregación local y calcularon el índice de segregación local denominado "variación del índice de disimilitud" (Dg) y el índice de segregación global propuesto por Silber (Ip). El estudio reveló que las mujeres y los hombres presentan un comportamiento diferente en el 
periodo considerado. La intensidad de la segregación se acentúa en función de la edad entre las mujeres, por lo que a mayor edad mayor especialización o confinamiento en determinadas ocupaciones-actividades. Sin embargo, los hombres de mayor edad pasan a estar más igualitariamente distribuidos en la escala ocupacional. El estudio muestra también que la disminución temporal observada en las diferencias por género para las distintas cohortes es debida a los cambios producidos en la estructura ocupacional. En consecuencia, se acentúan los niveles de concentración de las distintas cohortes de trabajadores en determinadas ocupaciones, configurando nichos laborales propios en función del sexo y de la edad del trabajador.

Esta investigación toma en cuenta la diferencia de género en los patrones de convivencia de los jóvenes en la actualidad, a través de una revisión de la literatura sobre este tema, analizaron los principales determinantes y presentaron las tendencias recientes en varios países (Adamopoulou, 2016).

La información obtenida a través de la aplicación del Análisis de Correspondencias Simples para este tipo de actividad demuestra la importancia de esta herramienta estadística cuando se aplica al ámbito de las ciencias sociales, validado con los resultados alcanzados para estas ciencias por otros investigadores (Batista y Sureda, 1987; Greenacre, 1993) y más recientemente (Beh, 2004).

La mayoría de los artesanos alcanzan el reconocimiento público como ya se mostró, después de su exitoso trabajo. La necesidad de realización lleva a los artesanos a ser emprendedores y a nunca dejar de trabajar, siempre motivados por la voluntad de hacer aquello que les gusta. Continúan su obra dedicándose a jornadas de trabajo que generalmente sobrepasan las dieciséis horas diarias. No obstante, estos emprendedores parecen trabajar más cuando existe alguna posibilidad de que sus esfuerzos personales influyan en el resultado del proceso que están ejecutando.

Fuentes y Sánchez (2016) en una similar investigación para profundizar en las diferencias, basándose en un estudio realizado en una amplia muestra de alumnado universitario, fuente de potenciales emprendedores. Los resultados indicaron una menor iniciativa emprendedora en las mujeres, que además consideran en mayor medida el temor al fracaso como un obstáculo a la hora de pensar en la creación de una empresa propia. También son distintos los atributos asociados por género a una mayor probabilidad de acometer este tipo de proyecto en el futuro (Fuentes y Sánchez, 2010). Estos autores consideran el emprendimiento como un motor de la innovación y el crecimiento. Recomiendan prestar especial atención a la implicación de la mujer en este ámbito por representar un grupo social de fuerza relevante en lo que a creación de empresas se refiere. Se necesita incidir en las diferencias de la figura de la potencial emprendedora, sus rasgos psicosociales, los estímulos y los principales obstáculos que encuentran las mujeres al iniciar una actividad empresarial.

\section{Acciones para lograr un espíritu emprendedor en la artesanía local de la provincia} Granma:

1. Fomentar entre los artesanos un ambiente de competitividad, enfocado en dos indicadores básicos, la calidad y autenticidad del producto, a través de concursos auspiciados por el ACAA. 
2. Celebrar anualmente un Festival de Artesanía en la provincia, cuya sede se seleccionará teniendo en cuenta el municipio que más se destaca en la producción y comercialización de sus obras.

3. Organizar talleres de capacitación, que involucren varias generaciones de artesanos, y fomentar la transmisión de saberes tradicionales.

4. Propiciar el abastecimiento regular de la materia prima necesaria para elaborar los productos, teniendo en cuenta las diversas opciones naturales que constituyen fuentes indispensables para elaborar las obras, sin descuidar el uso racional del medio ambiente.

5. Formación cultural de artesanos, de modo tal que sus creaciones sean un exponente fehaciente de las raíces culturales de la región.

6. Crear tiendas- talleres, con el objetivo de vender los productos y mostrar al cliente el proceso de realización de las artesanías.

7. Sensibilizar a los gobiernos locales en la importancia de desarrollar la producción y promoción artesanal.

8. Ubicar puntos de ventas estratégicos en las principales arterias de la provincia, con una adecuada decoración, que responda al tipo de venta que se realiza.

9. Impulsar campañas de publicidad a través de los medios de comunicación.

10. Buscar alianzas en zonas del Caribe que desarrollen la artesanía.

11. Realizar exposiciones artesanales en hoteles, restaurantes, escuelas, centros comerciales, entre otros.

12. Incentivar en niños y jóvenes la realización de actividades artesanales.

13. Armonizar criterios y establecer rutas probables de solución conjunta de los problemas que afectan la actividad.

14. Planificar y orientar las políticas y programas de desarrollo de la artesanía.

15. Fortalecer las condiciones de trabajo de los artesanos.

Tamayo y Leite (2015) consideran que la educación empresarial es el motor impulsor para inculcar la iniciativa emprendedora en los jóvenes cubanos, donde el talento florezca y permita llegar al entendimiento de que no todo el mundo puede convertirse en empresario. La innovación es el nombre del juego en los negocios, es difícil de lograr, porque no se trata solo de tener una buena idea. El capital intelectual y la gestión del conocimiento se convierten en los elementos claves para generar riquezas y obtener el éxito, estos conceptos forman el núcleo de una nueva teoría económica y de organización, que para lograrlas hay que ir más allá de la tecnología y el uso de la intuición.

\section{CONCLUSIÓN}

Luego de analizar desde el punto de vista teórico y conceptual la actividad artesanal en la provincia Granma y el papel del emprendimiento como elementos dinamizadores de las industrias culturales, se considera que la provincia Granma, particularmente los municipios de Bayamo y 
Manzanillo, poseen potencialidades para fomentar la producción artesanal. Además existe motivación por los jóvenes que fueron encuestados en la capacitación para hacer de sus productos obras cada vez más auténticas y representativas de la identidad local y nacional. Sin embargo es necesario implementar un plan de acciones que reduzca las debilidades que aún persisten y que imposibilitan el desarrollo artesanal de la provincia en toda su expresión. Es importante que todos los artesanos sean capacitados en el fenómeno del emprendimiento, creando empresas competitivas en el sector.

\section{REFERENCIAS BIBLIOGRÁFICA}

1. ADAMOPOULOU, E. Living arrangements of the youth: determinants and gender differences. Estudios de Economía Aplicada. ISSN: $1133-3197$ (Print) 1697-5731 (Online). Volumen 34-1: Economía de Género. Pág. 35-44. 2016.

2. ALDAZ, L., EGUÍA, B. Segregación laboral por género en España y en el País Vazco. Un análisis de cohortes. Estudios de Economía Aplicada. ISSN: 1133-3197 (Print) 1697-5731 (Online). Volumen 34-1: Enero 2016. Economía de Género. Pág. 133-154. 2016.

3. BANCO, S. Informe financiero. Publicado en: http://www.santander.com/.2012.

4. BATISTA, J.M., SUREDA J. Análisis de correspondencias y técnicas de clasificación: Su interés para la investigación en las ciencias sociales y del comportamiento. Revista Infancia y Aprendizaje, vol. 10, n.39-40, p.171-186. 1987.

5. BOUCHART, D. Artesanía y turismo. Publicado en: http://unesdoc.unesco.org/images/00 13/001362/. 2004.

6. BEH, E.J. Simple correspondence analysis: A bibliographic review. International. Statistic Review, vol. 72, n.2, p.257-284. 2004.

7. DRUCKER, F.P. Innovation and Entrepreneurship: Practices and Principles. New York: Harper \& Row.1985.

8. FUENTES, F., SÁNCHEZ, S. Análisis del perfil emprendedor: una perspectiva de género. Estudios de Economía Aplicada. Vol 28-3: 1-28. 2010.

9. GABRIEL, K.R. Biplot display of multivariate matrices with application to principal components analysis. Biometrika, n.58, p.453-467. 1971.

10. GREENACRE, M.J. Theory and Applications of Correspondence Analysis. London: Academic Press. 1984.

11. GREENACRE, M.J. Correspondence Analysis of Multivariate Categorical Data by Weighted Least-Squares. Biometrika, n.75, p.457-467. 1988.

12. GREENACRE, M.J. Biplots in correspondence analysis. Journal of Applied Statisties, Vol. 20, n.2, p.251-269. 1993.

13. GREENACRE, M.J., BLASIUS J. Correspondence Analysis in the Social Sciences, London: Academic Press. 1994.

14. HORKHEIMER, M. Y. Dialéctica de la razón. La Industria Cultural. Iluminismo como mistificación de las masas. Buenos Aires: Sudamericana. 1949.

15. - Infostat. Paquete Estadístico. Versión Libre. Universidad Nacional de Córdoba, 
Argentina. www.infostat.com.ar. 2013.

16. LEBART, L., MORINEAU A., WARWICK K.M. Multivariate Descriptive Statistical Analysis: Correspondence Analysis and Related Techniques for Large Matrices. New York: John Wiley \& Sons, Inc. 1984.

17. LEITE, F.E. O Fenômeno do Empreendedorismo. São Paulo: Saraiva, p. 560. 2012.

18. LEITE, F.E. Empreendedorismo, Inovação, Incubação de Empresas e Starups. Recife: Bagaço, p.551. 2015.

19. MCCLELLAND, C.D. The Achieving Society. New York: D. Van Nostrand Company. 1961.

20. MCCLELLAND, C.D. Human Motivation. Cambridge: Cambridge University Press, p.570. 1987.

21. NOVELO, V. Las artesanías en México. Tuxtla Gutiérrez, Chiapas. México: Gobierno del estado de . Chiapas, Instituto Chiapaneco de Cultura. 1993.

22. Observatorio Cultura y Economía. Las Industrias Culturales y Creativas. Su concepto y clasificación. Publicado en: http://culturayeconomia.org/blog/las-industrias-culturalesconcepto-clasificacion/. 2011.

23. PASTEURG, E. Artesanía prohibida. Primera edición. México: Instituto Nacional de Antropología e Historia, El Colegio de Michoacán, A.C., Universidad Autónoma de Yucatán. 2004.

24. PIEDRAS, E. ¿Cuánto vale la cultura? Contribución económica de las industrias protegidas por el derecho de autor en México. México: CONACULTA, CANIEN, SOGEN, SACM. México. 2004.

25. RAMOS, D. Los nuevos artesanos triunfan aplicando a sus productos una gestión empresarial moderna. Publicado en: http://www.emprendedores.es/gestion/gestion-empresaartesania/fortalezas-artesania. 2009.

26. SCHUMPETER, A.J. Capitalismo, Socialismo e Democracia. Tradução Carla Santos. Zahar Editores, Rio de janeiro, p. 98-211. 1984.

27. SCHUMPETER, A.J. Teoria do desenvolvimento econômico: uma investigação sobre lucros, capital, crédito, juro e o ciclo econômico. Tradução de Maria Sílvia Possas. São Paulo: Nova Cultura, p. 340. 1988.

28. TAMAYO, C., LEITE, E. Las tecnologías de la información y las comunicaciones como herramientas para la gestión del patrimonio cultural con una visión emprendedora. HOLOS, Año 31, Vol. 8:290-303. 2015.

29. THROSBYD. Panorámica del sector cultural y las Industrias culturales y creativas. Publicado en: http://culturayeconomia.org/blog/.2011.

30. VARGAS, J.G. Estrategias de la Industria Cultural Mexicana para el desarrollo regional del norte de Jalisco. El caso de la artesanía Huichol. 2013.

31. ZALLOR. Economía de la comunicación y la cultura. Madrid: Akal. 1988. 